\title{
Study on Human-structure Dynamic Interaction in Civil Engineering
}

\author{
Feng $\mathrm{GAO}^{1}$, Li Lin $\mathrm{CAO}^{2,}{ }^{*}$, Xing Hua $\mathrm{LI}^{3}$ \\ ${ }^{1}$ Department of State-owned Assets, Jiangsu University, Zhenjiang 212013, China. \\ ${ }^{2}$ Faculty of Civil Engineering and Mechanics, Jiangsu University, Zhenjiang 212013, China. \\ ${ }^{3}$ Jiangsu University Library, Zhenjiang 212013, China.
}

\begin{abstract}
The research of human-structure dynamic interaction are reviewed. Firstly, the influence of the crowd load on structural dynamic characteristics is introduced and the advantages and disadvantages of different crowd load models are analyzed. Then, discussing the influence of structural vibration on the human-induced load, especially the influence of different stiffness structures on the crowd load. Finally, questions about human-structure interaction that require further study are presented.
\end{abstract}

\section{Introduction}

In recent years, with the use of structural design, high-strength lightweight materials and construction technology, the structure of the building is gradually developing towards soft, long-span and low damping. The bearing capacity and deformation of the static performance can generally meet the requirement in the traditional design, but its dynamic performance, especially the excessive vibration of crowd excitation, may become a key problem restricting its application and development. The vibration caused by human activities is low frequency vibration, when the vertical natural frequency of this kind of structure is close to the frequency of the human activity, it is easy to cause resonance. This kind of vibration may exceed the tolerance limit of human comfort, cause panic in the psychology and seriously affect the performance of the structure. In the field of civil engineering, the perception of the human-structure interaction (HSI) was first derived from some phenomena found in some structural modal tests. Many studies show that people stay in long-span structure will affect the dynamic characteristics of the structure, and the vibration of long-span structure will have an impact on the excitation of crowd load, human-structure interaction is a new interdisciplinary scientific problem.

\section{The influence of crowd load on structural dynamic characteristics}

The crowd that stay in the building structure not only cause structural vibration, but also may change the modal characteristics. Therefore, how to consider the crowd stay in the structure is an urgent problem. Early on, Ebrahimpour regarded the crowd that stay in the structure as additional quality to consider its influence on the dynamic characteristics [1]. ATC design guide also uses a similar idea considering the influence on structural modal characteristics [2]. Obviously, the result of this treatment leads to a decrease in the natural frequency of the structure. However, Pimentel's research shows that presence of the crowd in remarkably crease structural damping [3]. Moreover, Brownjohn found that the single person that stay in concrete hollowed slab absorb more energy than structure itself[4]. Canada design code also consider the potential beneficial effects of crowd load on structural vibration response, it is proposed to increase the viscous damping ratio of the structure to $12 \%$ in the condition that crowd will stay in the structure[2]. The presence of people only regarded as the additional quality obviously cannot explain the research results given above.

In order to solve this problem, Rainer and Pernica pointed out the damped dynamic model of the crowd, this model can explain the phenomena that human occupants significantly increase the damping of the structure [5]. However, the additional mass model is widely accepted in a period, researchers believe that it can accurately predict the influence that human occupants change structural vibration frequency. Lenzing found that keep the single person weight over wood slab weight of more than 2 times, the frequency of the structure is not reduced, but a slight increase [6]. On 1997, Ellis and Ji carried out field dynamic characteristic test under two condition, the first was no load, the second considered the human occupants [7]. The second condition found a new mode of the stadium stands, and assume that the crowd as powered system with mass

*Corresponding author: Email: cll@ujs.edu.cn 
spring damper can better explain test result[7]. In 2002, Sachse et al. pointed out mathematical model of human occupants confirmed this assumption [8].In short, human occupants can increase structural damping, reduce fundamental frequency and add new modal mode. To accurately predict the effects of human occupants is of great significance for the economic design and safety evaluating of long-span structure. Therefore, a reasonable crowd load dynamic model must be established. In 1998, Griffin et al. used electro-hydraulic servo vibration table to test the contact force and displacement between the human body and the table of standing posture and sitting posture random vibration excitation, then obtained the basic frequency and damping ratio of the 60 tested human body[9]. In 2006, Liang et al. analyzed and compared the different degrees of freedom model based on the experimental data of vertical vibration of European and American sitting posture [10]. In 2011, Gao et al obtained the basic parameters of human vibration based on sitting posture of few Chinese people [11]. The above results are the basic human body parameters biomechanics field, mainly used in automobile, machinery and other fields of medicine, cannot directly apply to the civil engineering structure model of the human body, but useful research ideas for other researchers.

In the field of civil engineering, the simplified model of human body mainly consists of model with damping and without damping, and the model without damping is divided into discrete model and continuous model [12] (shown in Figure 1). Lenzing and Hothan studied the human single degree of freedom discrete model without damping, the lumped mass $m_{H}$ of the simplified human model was assumed to be equal to the total mass $m_{T}$ of the human body, and given the values of the stiffness and the natural frequency parameters of the human body model [13]. Later, Ji assumed that the upper and lower parts of the human body had different stiffness and mass, Falati assumed the stiffness and mass of the human body along the height distribution and established a continuous model without damping [14]. Foschi first studies the single degree of freedom damping model (Figure 2), obtained dynamic response based on the measured foot displacement time history curve and the calculation of single degree of freedom human body impact floor considering the human-structure interaction, the stiffness $k_{H}$ of the simplified kinetic model of human body is $40 \mathrm{kN} / \mathrm{m}$ and the viscous damping $c_{H}$ is $1.25 \mathrm{kNs} / \mathrm{m}$, but the premise of the study is that the lumped mass $m_{H}$ is equal to the total mass $m_{T}[15]$.The values of the natural frequencies $\left(f_{H}\right)$ and the damping ratio $\left(\xi_{H}\right)$ of the human body in the model are different from different scholars, and specific results are presented in table1.

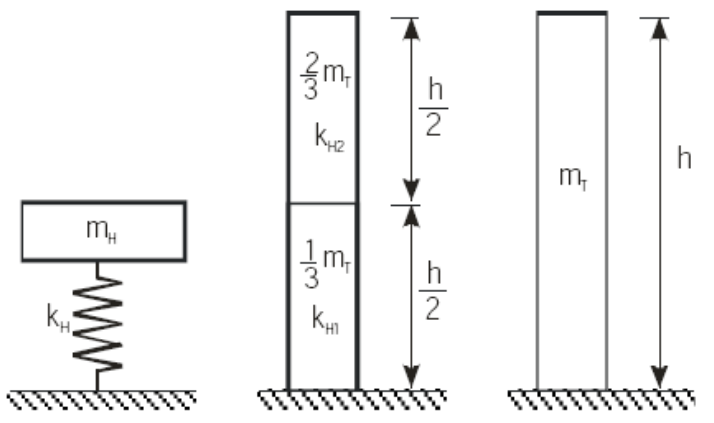

Figure 1 Simplified Model without Damping

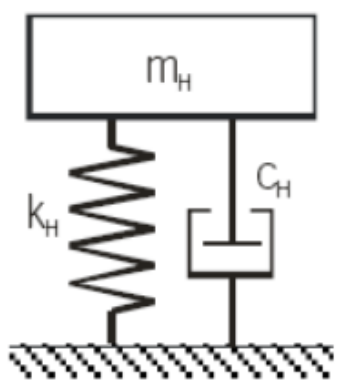

Figure 2 Single Degree of Freedom Model with Damping

Table 1 Comparison Between Numerical and Experimental Results

\begin{tabular}{cccc}
\multicolumn{5}{c}{ Table 1 Comparison Between Numerical and Experimental Results } \\
\hline \multirow{2}{*}{ Author } & $\begin{array}{c}\text { The natural frequency of } \\
\text { human body } f_{H}(\mathrm{~Hz})\end{array}$ & $\begin{array}{c}\text { The damping ratio of } \\
\text { human body } \xi_{H}\end{array}$ & $\begin{array}{c}\text { Lumped mass } \\
\text { value }\end{array}$ \\
\hline Falatiet al. & 10.43 & $45 \%-55 \%$ & $m_{H}=m_{T} / 3$ \\
Zhengand Brownjohn & 5.24 & $39 \%$ & $m_{H}=m_{T}$ \\
\hline
\end{tabular}

After the human body model is determined, the combined dynamic system of human-structure interaction can be analyzed. Sachse has established the system of two degrees of freedom dynamic human-structure coupling model (Figure 3a), and the natural frequencies, mode shapes, modal mass and damping ratio of the coupled system are analyzed in detail, the results of analysis showed that damped discrete model of the human-structure system with two degrees of freedom can better explain changes across the dynamic characteristics of structures with human occupants [16]. Zhou et al. uses a continuous model without damping to analyze the dynamic characteristics of a coupling system of human-structure interaction [17] (shown in Fig. 3b).

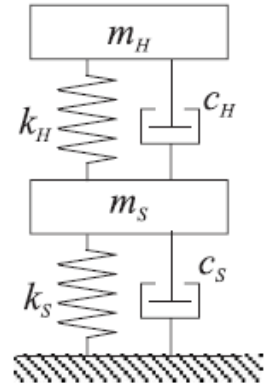

(a) Two Degrees of Freedom Discrete Model with Damping 


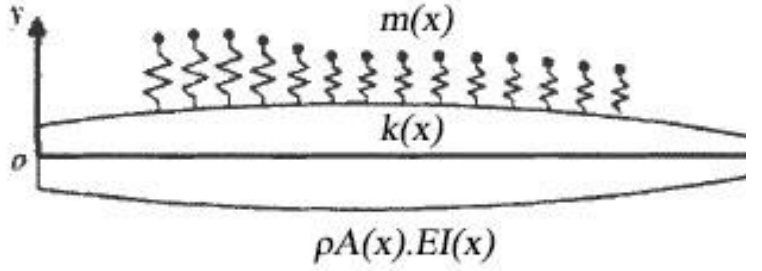

(b) Continuous Model without Damping

Figure 3 Human-structure Interaction Analysis Model

\section{Influence of structural vibration on human-induced load}

Effects of structural dynamic characteristics and vibration levels on crowd loads will happen when the vibration response of the structure is too large, the pedestrian adjust the pace and phase in order to improve walking comfort and synchronize with the structural vibration. In 1982, Ohlsson found that there were some differences in the measured walking load between the rigid ground and the long-span soft slab [18]. In 1988, Baumann and Bachmann indicated that the dynamic load coefficient measured on the rigid ground was increased by $10 \%$ compared to the $19 \mathrm{~m}$ long prestressed beam[19]. In 2002, Yao et al. pointed out that the dynamic load of jumping and running on the long-span floor is smaller than the rigid ground [20]. All the above studies show that the vibration of structure affects human-induced load, but there is some difference between the human activities in experiment and the actual structure, and the influence of individuals among the crowd is not considered. Therefore, it is of great significance to study the influence of long-span structure vibration with synchronous excitation on the human-induced load.

In recent years, the study of dynamic load of crowd excitation has attracted much attention. Research shows that the dynamic load generated by crowd excitation is much larger than that of single excitation, but the number of people and human-induced load is not a simple linear relationship. In some cases, people on the structure will do conscious or unconsciously synchronous activities. For example, the crowd in a rock ballroom will consciously synchronize with musical rhythm, which will have a greater impact on structural vibration. The involuntary, synchronous activity of the crowd may cause a violent vibration, which makes the structure uncomfortable and even insecure. It is an important research direction of the human-structure interaction that structural vibration is caused by the involuntary synchronization of the crowd. When the vibration of long-span structure reaches a certain extent, the crowd will adjust the walking pace and keep pace with the structural vibration. Grundmann et al. studied the synchronous modulation probability of vertical vibration between the crowd and structure, and proposed a method for calculating the synchronous modulation probability and vertical vibration acceleration [21]. So far, fewer research about synchronous modulation probability can be retrieved, it has important practical significance to deeply study synchronous modulation probability in the actual civil engineering structure.

\section{Conclusion and Prospect}

By reviewing the research of human-structure interaction, the following suggestions are put forward for the study of structural dynamic characteristics:

(1) Carrying out structural dynamic measurement. According to the experimental data, the parameters such as the lumped mass, the natural frequency of the body and the damping ratio of the human body are corrected and the crowd load model is refined.

(2) Considering the influence between individuals, developing the method for calculating vertical acceleration of structures based on synchronous modulation probability.

(3) At present, the study of human-structure interaction is more focused on the vertical vibration of structures, and the horizontal vibration of structures should be included in further study.

\section{Acknowledgements}

This study is financially supported by the National Natural Science Foundation of China (Grant No.51408267) and the Natural Science Foundation of the Jiangsu Higher Education Institutions of China (Grant No.14KJB560005) and Energy saving projects in Colleges and universities of Jiangsu Province (Grant No. JSSNZH2016301).

\section{References}

1. Ebrahimpour A, Sack R L, Vankleek $\mathrm{P}$ D. Computing crowd loads using a nonlinear equation of motion[C]. Proceeding of the 4th International Conference on Civil and Structural Engineering, 1989, 2: 47-52.

2. Allen, D E, Onysko D M, Murray T M. ATC Design guide 1: Minimizing floor vibration[S].1999, Redwood City, Canada, Applied Technology Council (ATC).

3. Pimentel R L, Waldron P. Validation of the numerical analysis of a pedestrian bridge for vibration serviceability applications[C]. Identification in Engineering Systems, Swansea, UK, 1996, Cromwell Press. 648-657.

4. Brownjohn, J M W. Energy dissipation from vibrating floor slabs due to human-structure interaction [J]. Shock and Vibration, 2001, $8(6): 315-23$.

5. Rainer J H, Pernica G. Vibration characteristics of a floor sample[C]. Ottawa, Canada: NRCC, Division of Building Research, 1985, NRCC-24298.

6. Lenzing H. Durch Menschen induzierteSchwingungen[D]. University Hannover, Hanover, Germany, 1988.

7. Ellis $\mathrm{B} \mathrm{R}, \mathrm{Ji}$ T. Human-structure interaction in vertical vibrations[C]. Proceedings of the ICE: Structures and Buildings, 1997, 122(1):1-9. 
8. Sachse R. The influence of human occupants on the dynamic properties of slender structures[D]. University of Sheffield, Sheffield, UK, 2002. [9] ANSI B. AISC 360-10-Specification for Structural Steel Buildings[J]. Chicago AISC, 2010.

9. Wei L, Griffin M J. Mathematical models for the apparent mass of the seated human body exposed to vertical vibration[J]. Journal of Sound and Vibration, 1998, 212: 855-74.

10. Liang C C, Chiang C F. A study on biodynamic models of seated human subjects exposed to vertical vibration[J]. International Journal of Industrial Ergonomics, 2006, 36(10): 869-890.

11. Gao J H, Hou Z C, He L, et al. Vertical vibration characteristics of seated human bodies and a biodynamic model with two degrees of freedom. SciChina Tech Sci, 2011, 54: 2776-2784

12. $\mathrm{Ji}, \mathrm{T}$. A continuous model for the vertical vibration of the human body in a standing position[C]. UK Informal Group Meeting on Human Response to Vibration, Silsoe, UK, 1995, 18-20.

13. Lenzing $H$. Durch Menschen induzierteSchwingungen[D]. Universität Hannover, Hanover, Germany, 1988.

14. Falati S. The contribution of non-structural components to the overall dynamic behaviour of concrete floor slabs[D]. University of Oxford, Oxford, UK, 1999.

15. Foschi R O, Neumann G A, Yao F, et al. Floor vibration due to occupant and reliability-based design guidelines[J]. Canadian Journal of Civil Engineering, 1995, 22(3):471-79.

16. Sachse R, Pavic A, Reynolds P. Parametric study of modal properties of damped two-degree-of-freedom crowd-structure dynamic systems[J]. Journal of Sound and Vibration, 2004, 274(3): 461-480.

17. Zhou D, Ji T. Free vibration of rectangular plates with continuously distributed spring-mass $[\mathrm{J}]$. International journal of solids and structures, 2006, 43(21): 6502-6520.

18. Ohlsson S. Floor vibrations and human discomfort[D]. Chalmers University of Technology, Gothenburg, Sweden, 1982.

19. Baumann K, Bachmann H. Man-induced dynamic loads and their influence on beam structures[R]. Zürich, Switzerland: Institute of Structural Engineering (IBK), 1988.

20. Yao S, Wright J, Pavic A, et al. Forces generated when bouncing or jumping on a flexible structure[C]. Proceedings of the International Conference on Noise and Vibration, Leuven, Belgium, 2002, 563-572.

21. Grundmann H, Kreuzinger $H$, Schneider $M$. Dynamic calculations of footbridges[J]. Bauingenieur, 1993, 68:215-225. 